\title{
TNF receptor 1 gene variant could explain failure of TNF-blocking drugs in multiple sclerosis
}

Drugs that block the activity of tumour necrosis factor (TNF) have been used successfully to treat various autoimmune conditions, yet have proved to be ineffective and even deleterious in patients with multiple sclerosis (MS). Reporting in Nature, Lars Fugger at the University of Oxford, UK, and his colleagues now suggest that a solution to this apparent paradox could lie in a variant in the TNFRSF1A gene, which encodes TNF receptor 1 (TNFR1).

"Over the last decade or so, we have been studying the functional effects of genetic risk factors for MS," says Fugger. "In addition, we have been working on a drug repositioning strategy, investigating the concept of using drugs already licensed for one disease for the treatment of another."

Fugger's team analysed genome-wide association study (GWAS) data from 1,853 patients with MS and 5,174 controls, along with whole-genome sequencing results obtained from 379 individuals as part of the 1000 Genomes project. Consistent with previous reports, they identified a strong association between MS and the rs1800693 single nucleotide polymorphism (SNP) in TNFRSF1A.

\section{$4 . . . \Delta 6$-TNFR1 ... was capable of binding to and thereby neutralizing TNF 77}

The researchers went on to examine how rs1800693 affects the function of TNFR1. They found that in individuals who carried this SNP, exon 6 was frequently spliced out of the TNFRSF1A mRNA, leading to production of a soluble form of TNFR1, termed $\triangle 6$-TNFR1, that was secreted from the cell instead of being sequestered in the Golgi apparatus.

Fugger and colleagues showed that the $\triangle 6$-TNFR1 protein isoform was capable of binding to and thereby neutralizing
TNF. “This risk effect mirrors the clinical experience with TNF blockers, whereby these drugs, whose properties are analogous to-although greater in magnitude than - those of $\Delta 6$-TNFR1, have been found to promote or exacerbate the disease," explains Fugger.

According to the researchers, these new findings reinforce the clinical utility of GWAS findings in conditions such as MS. "Such studies can enable the delineation of disease pathways, and as drugs targeting genetically dependent pathways are likely to have larger effects than any associated genetic variants, there is the potential to therapeutically skew these pathways towards disease resistance," concludes Fugger.

Heather Wood

Original article Gregory, A. P., Dendrou, C. A. et al. TNF receptor 1 genetic risk mirrors outcome of anti-TNF therapy in multiple sclerosis. Nature doi:10.1038/nature11307 